\title{
Real-time Triangulation of Molecular Surfaces
}

\author{
Joonghyun Ryu ${ }^{1}$, Rhohun Park ${ }^{1}$, \\ Jeongyeon $\mathrm{Seo}^{2}$, Chongmin Kim², Hyun Chan Lee ${ }^{3}$, and Deok-Soo Kim² \\ 1 Voronoi Diagram Research Center, Hanyang University \\ 17 Haengdang-dong, Seongdong-gu Seoul 133-791, Korea \\ \{jhryu, rhpark\}@voronoi.hanyang.ac.kr \\ 2 Department of Industrial Engineering, Hanyang University \\ 17 Haengdang-dong, Seongdong-gu Seoul 133-791, Korea \\ \{jyseo, cmkim\}@voronoi.hanyang.ac.kr, dskim@hanyang.ac.kr \\ 3 Department of Industrial Engineering, Hongik University \\ Sangsu-dong, 72-1. Mapo-gu, Seoul, Korea \\ hclee@wow.hongik.ac.kr
}

\begin{abstract}
Protein consists of a set of atoms. Given a protein, the molecular surface of the protein is defined with respect to a probe approximating a solvent molecule. This paper presents an efficient, as efficient as the realtime, algorithm to triangulate the blending surfaces which is the most critical subset of a molecular surface. For the quick evaluation of points on the surface, the proposed algorithm uses masks which are similar in their concepts to those in subdivision surfaces. More fundamentally, the proposed algorithm takes advantage of the concise representation of topology among atoms stored in the $\beta$-shape which is indeed used in the computation of the blending surface itself. Given blending surfaces and the corresponding $\beta$-shape, the proposed algorithm triangulates the blending surfaces in $O(c \cdot m)$ time in the worst case, where $m$ is the number of boundary atoms in the protein and $c$ is the number of point evaluations on a patch in the blending surface.
\end{abstract}

Key words: a protein, a molecular surface, $\beta$-shape, a Voronoi diagram of atoms

\section{Introduction}

It has been generally agreed that the structure of molecule is one of the most important factors which determines the functions of a molecule. Hence, studies have been conducted to analyze the structure of a molecule. Molecular surface is an important example of molecular structure.

Protein consists of a set of atoms where the atoms are usually modelled by spherical balls. Since a protein is usually solvated and the interaction between a protein and solvent molecules is important, we build a protein model in the solvent so that the interaction can be conveniently analyzed. A solvent molecule is usually approximated by a spherical ball, called a probe, which encloses a solvent molecule. This approximation is due to geometric as well as stochastic 
complexities of the system. Then, different types of surfaces, including a molecular surface, corresponding to the probe are defined on a protein [1-4].

Visualization of a molecular surface is important for studying various biological properties of molecules [4-6]. In particular, a fast visualization is preferred since there are usually many atoms in proteins. Ordinary proteins consist of thousands to hundreds of thousands atoms. Hence, the efficient triangulation of a molecular surface is critical to facilitate a fast visualization. Besides, the surface area and volume, which are important mass properties for understanding the characteristics, of a molecule can be also easily calculated from the triangulation of the surface [7].

This paper presents an algorithm for efficiently triangulating the blending surfaces of a protein which is the important part of the molecular surface of the protein. We consider that the blending surfaces themselves are computed as a preprocessing via the $\beta$-shape of a protein corresponding to the probe $[8,9]$.

\section{Related Works}

Richards was the first who defined the molecular surface of protein [2]. Since then, several computational studies of the surfaces on a protein have been conducted. Connolly computed the molecular surface of a protein to calculate the protein volume, electrostatic potential, and interface surfaces between molecules [3]. Connolly also presented an analytic representation of a molecular surface [10] where he pointed out that a molecular surface consists of three types of patches: a convex spherical patch, a saddle-shaped toroidal patch and a concave spherical patch. Later, Connolly discussed a triangulation of a molecular surface [7].

Sanner et al. provided a more efficient algorithm for a molecular surface which uses a reduced surface of a molecule which can be computed from a binary spatial division tree [11]. It is very interesting to find that the reduced surface is indeed equivalent, in its concept, to an instance of the $\beta$-shape. Varshney et al. presented an algorithm based on a spatial grid which facilitates a relatively efficient neighbor search among atoms [12]. Bajaj et al. presented a trimmed NURBS (Non-Uniform Rational B-Spline) representation of a molecular surface so that a standard graphics library such as OpenGL can be conveniently used [13]. In this work, they used the power diagram of atoms for a neighbor search. Later, they also discussed a condition for re-computing molecular surfaces for the probes of varying sizes without re-computing the power diagram [14].

Edelsbrunner et al. introduced the concept of a molecular skin surface, which is the implicit surface defined by the envelope of a family of infinitely many spheres controlled by a finite collection of weighted points [15]. Different from other approaches, the skin surface is tangent continuous and does not selfintersect. There are several works on the triangulations of a molecular skin surface $[16,17]$.

In this paper, we present a fast, as fast as a realtime, algorithm for triangulating blending surfaces in a molecular surface of a protein. We consider 
that blending surfaces in a molecular surface are available, as a preprocessing, via the $\beta$-shape of atoms which is again computed from the quasi-triangulation of atoms [18-20]. Note that a quasi-triangulation is the dual structure of the Voronoi diagram of atoms.

\section{Surface Types on a Molecule}

Let $A=\left\{a_{1}, a_{2}, \ldots, a_{n}\right\}$ be a finite set of three-dimensional spherical atoms $a_{i}=\left\{x|| x-c_{i} \mid \leq r_{i}\right\}$ where $c_{i}$ and $r_{i}$ are the center and van der Waals radius of $a_{i}$, respectively. A protein, a DNA, or a RNA may be considered an example of the set $A$.

Definition 1. Let $\mathcal{V}(A)=\left\{x \in \mathbb{R}^{3} \mid x \subset \bigcup a_{i} \in A\right\}$. Then, the boundary $\partial \mathcal{V}(A)$ of $\mathcal{V}(A)$ is the van der Waals surface of a molecule $A$.

Let a probe $p=\left(c_{p}, r_{p}\right)$ be an open ball where $c_{p}$ and $r_{p}$ are the center and the radius of the probe. Consider the union of all possible empty probes in $\mathbb{R}^{3}$. Then, we can define a molecular surface of $A$ by the complement of the union and $\mathcal{V}(A)$.

Definition 2. $M S_{p}(A)=\left\{\partial\left(\mathbb{R}^{3}-\bigcup p\right) \mid p \bigcap \mathcal{V}(A)=\emptyset\right\}$ is the molecular surface of a molecule $A$ for a given probe $p$.

$M S_{p}(A)$ consist of points on the van der Waals surface of atoms, called a solvent contact surface SCS and other points from the surface of a probe, called a reentrant surface $R S$. $R S$ consists of two types of surface regions: a link blending surface and a rolling blending surface. A link blending surface is defined when a probe is located on the top of a triplet of atoms and a rolling blending surface is defined when a probe rolls over a pair of atoms $[10,12,8]$. In this paper, we present a realtime algorithm for triangulating all blending surfaces in $M S_{p}(A)$.

A link blending surface consists of a set of spherical reentrant surface patches, called link patches, from the probe boundaries which are on the top of nearby three atoms. A rolling blending surface consists of a set of toroidal reentrant surface patches, called rolling patches, which are defined by a set of empty probes between two nearby atoms. Examples of link patches and rolling patches are shown in Fig. 1 (a).

\section{$4 \beta$-shape for Blending Surfaces}

A $\beta$-shape is a generalization of the well-known theory of $\alpha$-shape which is initially proposed in [21] and later extended to the concept in 3D by Edelsbrunner et al. [22]. Since the initial proposal of $\alpha$-shape is not able to incorporate the weights of points properly, the weighted $\alpha$-shape was proposed [23]. However, the weighted $\alpha$-shape is not very convenient to provide a correct answer efficiently to general queries on the proximity among non-intersecting atoms in Euclidean distance metric because the weighted $\alpha$-shape is based on the power distance 


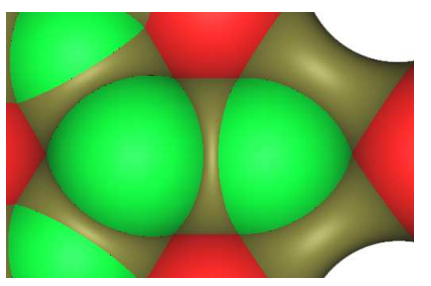

(a)

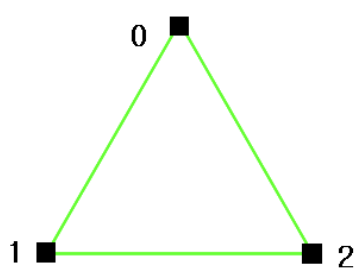

(b)

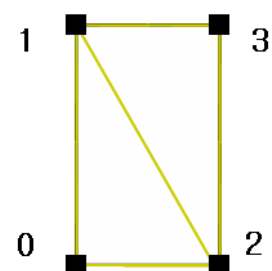

(c)

Fig. 1. An example of a molecular surface. (a) Examples of link patches and rolling patches, (b) the topology of a triangle on a link patch of the crudest resolution(depth 0), and (c) the topology of two triangles on a rolling patch of the crudest resolution(depth $0)$

metric [24]. To fully incorporate the variation of atom sizes, a theory of $\beta$-shape was devised. The $\beta$-shape in $3 \mathrm{D}$ is a polytope bounded by vertices, edges and triangles as an $\alpha$-shape is. Given an atom set $A$ corresponding to a protein, a $\beta$-shape $\mathcal{S}_{\beta}(A)$ for $A$ by a particular $\beta$-probe, where its radius is $\beta$, is defined as adopted from [24].

Definition 3. Let $p$ be a $\beta$-probe corresponding to a particular value of $\beta, 0 \leq$ $\beta \leq \infty$, and located at a particular location in $\mathbb{R}^{3}$. Let $\widetilde{A}(p)=\{a \in A \mid p \cap A=$ $\emptyset, a \cap \partial(p) \neq \emptyset\}$ and $\widetilde{C}(p)=\{c \mid a=(c, r) \in \widetilde{A}(p)\}$. Suppose $\triangle_{p}$ is the convex combination of elements in $\widetilde{C}(p)$. Then, the $\beta$-shape $\mathcal{S}_{\beta}(A)$ of $A$ is defined as a polytope bounded by a set $\bigcup \triangle_{p}$, for all possible $p$ in the space.

Each blending patch in a molecular surface $M S_{p}(A)$ can be identified by referring to the edges and the triangular faces in $\partial \mathcal{S}_{\beta}(A)$. It is known that the number of edges and triangular faces in $\partial \mathcal{S}_{\beta}(A)$ is bounded by $O(n)$ for molecules in the worst case where $n=|A|[8,25,12]$. Therefore, it is obvious that the blending surfaces in a molecular surface $M S_{p}(A)$ can be computed in $O(n)$ if each edge or triangular face on $\partial \mathcal{S}_{\beta}(A)$ independently defines a rolling or a link patch, respectively. However, it is not the case in the molecular surface since intersections may often exist among link patches. Surprisingly, it is shown that, even in this case, the blending surfaces in a molecular surface can be correctly computed in $O(n)$ time in the worst case if the $\beta$-shape is properly used [9].

\section{Triangulation of Blending Surfaces}

Visualization of a molecular surface is important for studying various biological properties of molecules [4-6]. For this purpose, the efficient computation of both the mathematical representation and the triangulation of the surfaces are critical since the number of atoms in molecules is usually significant [26-28]. In this section, we discuss how to triangulate the blending surfaces efficiently assuming that blending surfaces are available. 


\subsection{Triangulation of a Link Patch}

Once a link patch and a rolling patch are computed, we need to triangulate the patches in order to render the protein. In this case, the evaluation of sample points on a patch is necessary for the triangulation of the patch.

A link patch is defined when a probe is on the top of three nearby atoms. If we assume that a link patch does not intersect any other link patch, an initial link patch $\lambda^{I}$ is a spherical triangle where each boundary edge of the patch is an arc on a great circle of probe boundary $\partial p$.

Let $c_{i}, i=0,1$ and 2 , be the contact points on three atoms where a probe $p$ touches the atoms. Hence, $c_{i}$ 's are the vertices of $\lambda^{I}$. Let $b_{i}^{I}, i=0,1$ and 2 , be three $\operatorname{arcs}$ of $\lambda^{I}$ defined by the vertices $c_{i}$ and a probe center $c_{p}$. Then, sample points on $b_{i}^{I}$ can be evaluated with a uniform distribution (in the distance point of view between consecutive evaluations) by recursive bisections on $b_{i}^{I}$. Suppose that the edge $b_{0}^{I}$ is defined between $c_{0}$ and $c_{1}$ and let $m_{3}$ be the mid point on the edge. Then, $m_{3}$ can be obtained by the following equation.

$$
m_{3}=c_{p}+\overrightarrow{u_{01}} \times r_{p}
$$

where $r_{p}$ is a radius of a probe $p$ and $\overrightarrow{u_{01}}$ is the unit vector which bisects the angle between $\overrightarrow{c_{p} c_{0}}$ and $\overrightarrow{c_{p} c_{1}}$. Other sample points on the edge can be evaluated by recursively applying Eq. (1). Similar calculations can be applied to other edges of $b_{1}^{I}$ and $b_{2}^{I}$.

Once sample points on the boundary arcs are evaluated, we can also evaluate sample points in the interior of a link patch via Eq. (1). Fig. 2 shows schematic diagrams of two examples for evaluating sample points on a link patch. The black rectangles in this figure represent three contact points $c_{i}, i=0,1$, and 2 and the black dots represent the uniformly evaluated sample points. All sample points in Fig. 2 (a) are on the boundary edge while sample points in Fig. 2 (b) are both on the boundary and in the interior.

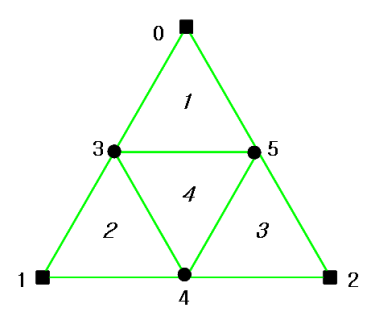

(a)

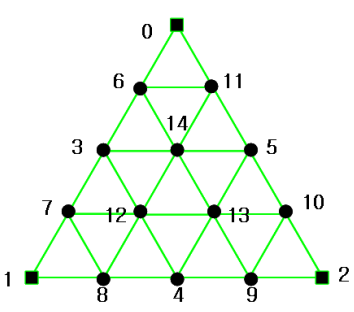

(b)

Fig. 2. Sampling points on a link patch via mid point calculation. (a) an example for depth 1 , (b) an example for depth 2 
Definition 4. Consider a link patch $\lambda$ with three contact points $c_{i}, i=0,1$, and 2. Let $D^{L}$ be the sampling depth of a link patch $\lambda$ where $4^{D^{L}}$ is the number of triangles on $\lambda$. The triangles are obtained by recursive subdivisions from the initial triangle defined by $c_{i}, i=0,1$, and 2 .

Fig. 1 (b) shows an example of a link patch with sampling depth $D^{L}=0$. Fig. 2 (a) and (b) show the sampling depths of 1 and 2, respectively. Note that the link patches in Fig. 2 (a) and (b) consist of 4 and 16 triangles. Given three contact points, we can evaluate additional points between every pair of sample points and the order of point evaluations can be determined a priori.

Lemma 1. Given a sampling depth $D^{L}$ and three contact points of a link patch, uniformly distributed sample points on the patch can be evaluated in an order determined a priori.

Proof. Given two sample points $v_{i}$ and $v_{j}$ in an initial link patch $\lambda^{I}$, a new sample point between $v_{i}$ and $v_{j}$ can be computed via Eq. (1) regardless $v_{i}$ and $v_{j}$ are on the boundary edges of $\lambda^{I}$ or not. Hence, we can evaluate all sample points necessary for a given sampling depth in two steps as follows. First, evaluate a new sample point between old sample points on each boundary edge of a link patch. Then, evaluate new sample points in the interior of a link patch by using sample points on boundary edge. Therefore, if three contact points have a consistent order in their sequence, new sample points are evaluated in consistent order.

The numbers attached near sample points in Fig. 2 represent the orders that the points themselves are evaluated in case that three contact points are given in a counter-clockwise order. To evaluate sample points in consistent order and triangulate the evaluated sample points, we maintain two types of masks: an edge index mask and a triangle index mask.

Definition 5. Suppose that $D^{L}=i$. An edge index mask $E_{i}^{L}$ is a set of a pair of integers where each pair of integers represents two indices for two sample points on a link patch.

An edge index mask of a link patch with sampling depth $D^{L}=0$ is $E_{0}^{L}=\{(0,1),(1,2),(2,0)\}$ if three indices of three contact points are 0,1 and 2 . Similarly, $E_{1}^{L}=\{(0,3),(3,1),(1,4),(4,2),(2,5),(5,0),(3,4),(4,5),(5,3)\}$ is an edge index mask for a link patch with $D^{L}=1$ (See Fig. 2 (a)). Each edge in an edge index mask corresponds to a new sample point to be evaluated. For example, $v_{3}$ is computed by referring to an edge $(0,1) \in E_{0}^{L}$. Note that an edge index mask is invariant for any link patch with same sampling depth.

Definition 6. Suppose that $D^{L}=i$. A triangle index mask $T_{i}^{L}$ is a set of a triplet of integers where each triplet of integers represents three vertices of each triangle defined by sample points on a link patch.

Hence, a triangle index mask of a link patch with $D^{L}=0$ is $T_{0}^{L}=\{(0,1,2)\}$ and similarly, $T_{1}^{L}=\{(0,3,5),(1,4,3),(2,5,4),(3,4,5)\}$ is a triangle index mask 
for a link patch with $D^{L}=1$. Once a triangle index mask $T_{i}^{L}$ is obtained for a given sampling depth $D^{L}=i$, the triangulation of sample points in all link patches is completed because a triangle index mask $T_{i}^{L}$ is invariant for any link patch with $D^{L}=i$.

Theorem 1. Given an edge index mask $E_{i-1}^{L}$ and a triangle index mask $T_{i-1}^{L}$, an edge index mask $E_{i}^{L}$ and a triangle index mask $T_{i}^{L}$ can be obtained by splitting each edge in $E_{i-1}^{L}$ into two edges and subdividing each triangle in $T_{i-1}^{L}$ into four triangles.

Proof. $E_{i}^{L}$ consist of two types of edges: the boundary edges $E_{i}^{B}$ or the interior edges $E_{i}^{I}$ of a link patch with $D^{L}=i . E_{i}^{B}$ can be obtained by splitting each edge in $E_{i-1}^{B}$ into two contagious edges where each edge can be split into two edges by inserting a new vertex between two vertices of the edge. $E_{i}^{I}$ can be obtained by splitting each edge in $E_{i-1}^{I}$ or by connecting each pair of new inserted vertices which can be identified by referring to each triangle $t \in T_{i-1}^{L}$.

Once $E_{i}^{L}$ is obtained, $T_{i}^{L}$ can be constructed by subdividing each triangle $t \in T_{i-1}^{L}$ into four smaller triangles. The subdivision can be done by referring to three new vertices in $E_{i}^{I}$ which are inserted to each edge of $t \in T_{i-1}^{L}$.

Therefore, $E_{i}^{L}$ and $T_{i}^{L}$ can be computed by repeatedly applying the procedure in Theorem 1 to $E_{0}^{L}$ and $T_{0}^{L}$. Given $E_{i-1}^{L}, T_{i}^{L}$ and three contact points, all the necessary sample points of all link patches can be evaluated by referring to $E_{i-1}^{L}$ and all link patches can be triangulated by referring to $T_{i}^{L}$. The order that each triangle in $T_{i}^{L}$ is generated can be identified by referring to the order of three vertices of $t \in T_{i-1}^{L}$ as shown in Fig. 2 (a). Note that $E_{i-1}^{L}$ and $T_{i}^{L}$ are invariant for a given sampling depth $D^{L}=i$ and the computation for generating $E_{i-1}^{L}$ and $T_{i}^{L}$ is needed only once.

\subsection{Triangulation of a Rolling Patch}

A rolling patch is defined when a probe rolls over two nearby atoms. Hence, sample points on a rolling patch can be determined by a set of sample probes (probe positions) which come in tangential contact with two atoms. The locus of the probes defining a rolling patch is either an arc or a complete circle. Therefore, sample probes for determining sample points on a rolling patch can be computed via Eq. (1). Given a particular probe from sample probes, sample points defined by the probe can be evaluated by using Eq. (1) due to the following property.

Property 1. Given a particular probe $p$ which is in tangential contact with two nearby atoms, sample points of the rolling patch defined by $p$ are on an arc of a great circle of the probe boundary $\partial p$.

Fig. 3 shows schematic diagrams of two examples for evaluating sample points of a rolling patch. Black rectangles and dots are sample points on the sharing boundary edges with adjacent link patches and each column of white dots represents sample points evaluated from each sample probe. 
In this section, we assume that a rolling patch is partial where the locus of a rolling patch is a circular arc. Note that a rolling patch can be complete; i.e., a rolling patch does not have adjacent link patches and its locus is a complete circle [8]. In this case, if we divide the rolling patch into two sheet of patches, we can handle these two patches as if they are two separate partial rolling patches.

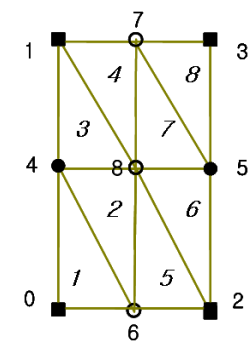

(a)

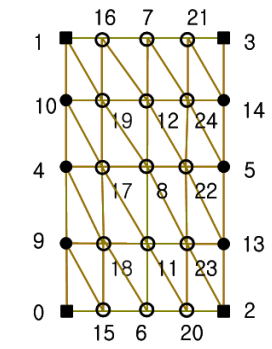

(b)

Fig. 3. Sampling points on a rolling patch. (a) an example for depth 1, (b) an example for depth 2

Definition 7. Consider a rolling patch $\gamma$ with four corner points. Let $D^{R}$ be the sampling depth of a rolling patch $\gamma$ where $2 \cdot 4^{D^{R}}$ is the number of triangles on $\gamma$. The triangles are obtained by recursive subdivisions from initial two triangles defined by four corner points.

Fig. 1 (c) shows an example of a rolling patch with sampling depth $D^{R}=0$ where four corner points are from two adjacent link patches. Fig. 3 (a) and (b) show the rolling patches with the sampling depths 1 and 2, respectively. Note that the rolling patches in Fig. 3 (a) and (b) consist of 8 and 32 triangles. Given four corner points, we can evaluate additional sample points between every pair of sample points and on the boundary of additional sample probes. The order of point evaluations can be determined a priori.

Lemma 2. Given a sampling depth and four corner points of a rolling patch, all of the necessary points uniformly distributed on the patch can be evaluated in consistent order.

Proof. Each sample point on a rolling patch has its corresponding sample probe $p$ where $p$ is in tangential contact with two nearby atoms. Hence, we can evaluate all sample points necessary for a given sampling depth in two steps. First, evaluate new sample points between old sample points which correspond to old sample probes. Then, compute new sample probes between old sample probes and evaluate new sample points on the boundaries of new sample probes. Therefore, if four corner points of a rolling patch have a consistent order in their sequence, new sample points are evaluated in consistent order. 
The number attached near sample points in Fig. 3 represent the order that the points are evaluated in case that four corner points are given in the order shown in Fig. 1 (c). To evaluate sample points in consistent order and triangulate the evaluated sample points, we maintain two types of index masks: an edge index mask and a triangle index mask.

Definition 8. Suppose that $D^{R}=i$. An edge index mask $E_{i}^{R}$ is a set of a pair of integers where each pair of integers represents two indices for two contagious sample points on each sample probe defining a rolling patch.

An edge index mask of a rolling patch with sampling depth $D^{R}=0$ is $E_{0}^{R}=\{(0,1),(2,3)\}$ if the indices of four corner points are 0, 1, 2 and 3. Similarly, $E_{1}^{R}=\{(0,4),(4,1),(6,8),(8,7),(2,5),(5,3)\}$ is an edge index mask for a rolling patch with $D^{R}=1$ (See Fig. $3(\mathrm{a})$ ). Each edge in an edge index mask corresponds to a new sample point to be evaluated. For example, $v_{8}$ is computed by referring to an edge $(6,7) \in E_{0}^{R}$. Note that an edge index mask is invariant for any rolling patch with same sampling depth.

Definition 9. Suppose that $D^{R}=i$. A triangle index mask $T_{i}^{R}$ is a set of a triplet of integers where each triplet of integers represents three vertices of each triangle defined by sample points on a rolling patch.

Hence, a triangle index mask of a rolling patch with $D^{R}=0$ is $T_{0}^{R}=$ $\{(0,2,1),(1,2,3)\}$ and similarly, $T_{1}^{R}=\{(0,6,4),(4,6,8), \cdots,(8,5,7),(7,5,3)\}$ is a triangle index mask for a rolling patch with $D^{R}=1$. Once a triangle index mask $T_{i}^{R}$ is obtained for a given sampling depth $D^{R}=i$, the triangulation of sample points in all rolling patches is completed because a triangle index mask is invariant for any rolling patch with $D^{R}=i$.

Theorem 2. Given an edge index mask $E_{i-1}^{R}$ and a triangle index mask $T_{i-1}^{R}$, an edge index mask $E_{i}^{R}$ and a triangle index mask $T_{i}^{R}$ can be obtained by splitting each edge in $E_{i-1}^{R}$ into two edges and inserting new edge list corresponding to new sample probes.

Proof. Sample points corresponding to the indices of $E_{i}^{R}$ belong to one of $\left(2^{i}+1\right)$ groups where each group of sample points is defined by a sample probe in $P_{i}^{R}$. Sample probes in $P_{i}^{R}$ for $E_{i}^{R}$ consist of two types of probes: old sample probes for $E_{i-1}^{R}$ and new sample probes for $E_{i}^{R}$. Hence, $E_{i}^{R}$ can be obtained in two steps. First, split each edge in $E_{i-1}^{R}$ into two contagious edges by inserting a vertex between two vertices of the edge. Then, insert new groups of edges between old groups of edges which correspond to $P_{i-1}^{R}$.

Once $E_{i}^{R}$ is obtained, $T_{i}^{R}$ can be constructed by indexing each triplet of indices in two contagious groups of edges in $E_{i}^{R}$ as three vertices for each triangle in $T_{i}^{R}$.

Therefore, given a sampling depth $D^{R}=i$ of a rolling patch, $E_{i}^{R}$ and $T_{i}^{R}$ can be computed by repeatedly applying the procedure in Theorem 2 to $E_{0}^{R}$ and $T_{0}^{R}$. Given $E_{i-1}^{R}$ and $T_{i}^{R}$ and four corner points, all the necessary sample points of 
all rolling patches can be evaluated by computing sample probes and referring to $E_{i-1}^{R}$ and all rolling patches can be triangulated by referring to $T_{i}^{R}$.

The order that each triangle in $T_{i}^{R}$ is generated can be identified by referring to each triplet of vertices in two contagious groups of edges in $E_{i}^{R}$ as shown in Fig. 3 (a). Note that $E_{i-1}^{R}$ and $T_{i}^{R}$ are invariant for a given sampling depth $D^{R}=i$ and the computation for generating $E_{i-1}^{R}$ and $T_{i}^{R}$ is needed only once.

\subsection{Handling of Intersections among Link Patches}

Intersections may occur among link patches either at the boundary or in the interior of the patches $[13,14,8]$. When intersections occurs, we need to modify the triangulation scheme discussed in Sec. 5.1 and 5.2 in order to obtain the water-tight triangular mesh. Consider two adjacent link patches intersect each other and in-between rolling patch self-intersects. In this case, if we apply same sampling depth to two link patches and two disconnected components of a selfintersecting rolling patch, we will necessarily have the gap at the boundary as shown in Fig. 4 (a). In this figure, shown are two adjacent link pathes of depth $D^{L}=2$ and an in-between rolling patch with two disconnected components of $D^{R}=2$.

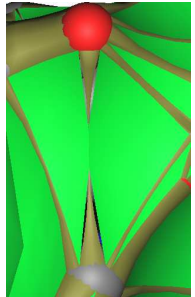

(a)

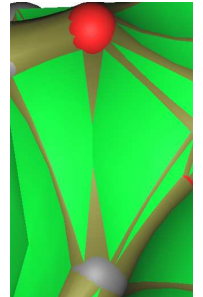

(b)

Fig. 4. An example for the triangulation of the blending patches. (a) the triangular mesh with a gap between the boundary with depth $D^{L}=D^{R}=2$ (b) a water-tight triangular mesh with depth $D^{L}=2$ and $D^{R}=0$

Fig. 4 (b) shows that the gap between a link patch and a rolling patch in Fig. 4 (a) can be filled by applying sampling depths $D^{R}=0$ and $D^{L}=2$ to a rolling patch and a link patch, respectively, based on the following lemma.

Lemma 3. Suppose we apply $D^{L}=i$ and $D^{R}=i-2$ to a link patch and a self-intersecting rolling patch. Then, we can fill the gap between a link patch and a rolling patch by using $2^{i-1}-1$ points to represent the set of trimming arc segments. 


\section{Discussion and Conclusion}

We tested the proposed algorithm using 50 protein models available from Protein Data Bank (PDB) [27]. Fig. 5 (a) shows a protein model (PDB ID: 1IZH) from PDB which is a inhibitor of HIV protease and consists of 1570 atoms. Fig. 5 (b) and (c) illustrate the molecular surface of a protein in Fig. 5 (a) corresponding to a water molecule and its blending surfaces, respectively.

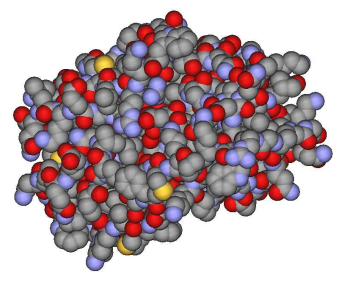

(a)

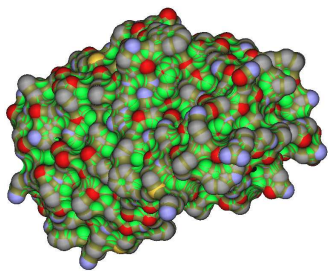

(b)

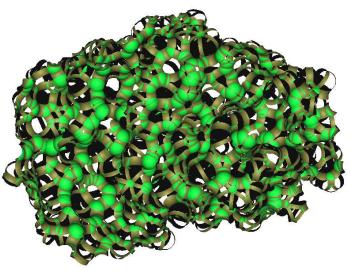

(c)

Fig. 5. An example of a molecular surface. (a) a protein model (Inhibitor of HIV protease, PDB ID: 1IZH), (b) a molecular surface of a protein in (a) corresponding to a water molecule and (c) blending surfaces in the molecular surface of (b)

Fig. 6 (a) shows the time statistics for triangulating blending surfaces in a molecular surface for each sampling depth. X-axis of the graph in Fig. 6 (a) represents the number of boundary atoms in each protein and Y-axis represents time for triangulating blending surfaces in a molecular surface corresponding to a water molecule.

For each fixed sampling depth, the times to triangle blending surfaces show a strong linear pattern with respect to the number of boundary atoms in a protein. Note that the computation for generating an edge mask and a face mask is needed only once for a fixed sampling depth. Hence, once the proposed algorithm generates edge and triangle index masks for each sampling depth, the algorithm can triangulate all blending patches just by evaluating necessary sample points of the blending patches. Fig. 6 (b) shows the number of triangles in blending surfaces of same protein data referred in the graph of Fig. 6 (a).

This paper presents an algorithm to triangulate the blending surfaces in a molecular surface of a protein efficiently. The number of link patches and rolling patches in blending surfaces of a molecular surface is bounded by the number of the boundary atoms in a protein. Given the blending surfaces and its corresponding $\beta$-shape, the blending surfaces can be triangulated in $O(c \cdot m)$ in the worst case, where $m$ is the number of boundary atoms in the protein and $c$ is the number of point evaluations on a patch in the blending surface. 


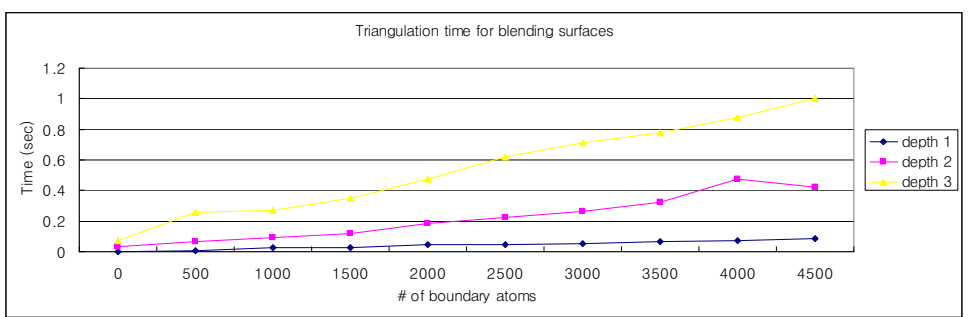

(a)

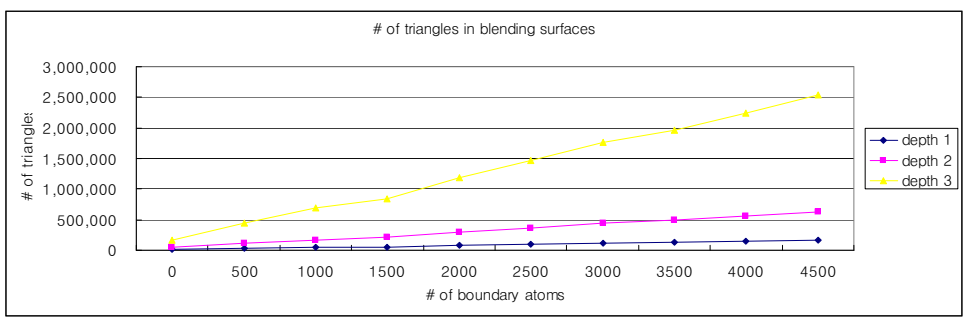

(b)

Fig. 6. Triangulation time and the number of triangles of blending surfaces. (a) triangulation time for blending surfaces in each protein data, (b) the number of triangles in blending surfaces of each protein data used in (a)

\section{Acknowledgments.}

Joonghyun Ryu and Rhohun Park were supported by the Creative Research Initiatives and Jeongyeon Seo, Chongmin Kim and Deok-Soo Kim were supported by BK21. Hyun Chan Lee was supported by the Basic Research Program of the Korea Science \& Engineering Foundation (No. R01-2006-000-10327-0).

\section{References}

1. Lee, B., Richards, F.M.: The interpretation of protein structures: Estimation of static accessibility. Journal of Molecular Biology 55 (1971) 379-400

2. Richards, F.M.: Areas, volumes, packing, and protein structure. Annual Review of Biophysics and Bioengineering 6 (1977) 151-176

3. Connolly, M.L.: Solvent-accessible surfaces of proteins and nucleic acids. Science 221 (1983) 709-713

4. Connolly, M.L.: Molecular surfaces: A review. Network Science (1996) http://www.netsci.org/Science/Compchem/feature14.html.

5. Leach, A.R.: Molecular Modelling: Principles and Applications. Prentice Hall (2001)

6. Bourne, P.E., Addess, K.J., Bluhm, W.F., Chen, L., Deshpande, N., Feng, Z., Fleri, W., Green, R., Merino-Ott, J.C., Townsend-Merino, W., Weissig, H., Westbrook, J., Berman, H.M.: The distribution and query systems of the rcsb protein data bank. Nucleic Acids Research 32 (2004) D223-D225 
7. Connolly, M.L.: Molecular surface triangulation. Journal of Applied Crystallography 18 (1985) 499-505

8. Ryu, J., Park, R., Kim, D.S.: Molecular surfaces on proteins via beta shapes. Computer-Aided Design (in press) (2007)

9. Ryu, J., Park, R., Cho, Y., Seo, J., Kim, D.S.: beta-shape based computation of blending surfaces on a molecule. In: The 4th ISVD International Symposium on Voronoi Diagrams in Science and Engineering. Volume (accepted). (2007)

10. Connolly, M.L.: Analytical molecular surface calculation. Journal of Applied Crystallography 16 (1983) 548-558

11. Sanner, M., Olson, A.J., Spehner, J.C.: Reduced surface: An efficient way to compute molecular surfaces. Biopolymers 38 (1996) 305-320

12. Varshney, A., Brooks, Jr., W.V.W.: Computing smooth molecular surfaces. IEEE Computer Graphics and Applications 14 (September 1994) 19-25

13. Bajaj, C.L., Lee, H.Y., Merkert, R., Pascucci, V.: NURBS based b-rep models for macromolecules and their properties. In: Proceedings of the 4th Symposium on Solid Modeling and Applications. (1997) 217-228

14. Bajaj, C.L., Pascucci, V., Shamir, A., Holt, R.J., Netravali, A.N.: Dynamic maintenance and visualization of molecular surfaces. Discrete Applied Mathematics 127(1) (2003) 23-51

15. Edelsbrunner, H.: Deformable smooth surface design. Discrete \& Computational Geometry 21 (1999) 87-115

16. Cheng, H.L., Dey, T.K., Edelsbrunner, H., Sullivan, J.M.: Dynamic skin triangulation. Discrete \& Computational Geometry 25(4) (2001) 525-568

17. Cheng, H.L., Shi, X.: Guaranteed quality triangulation of molecular skin surfaces. In: [Vis 2004] IEEE Visualization. (October 10-15, Austin, Texas, USA 2004) 481488

18. Kim, D.S., Cho, Y., Kim, D.: Euclidean Voronoi diagram of 3D balls and its computation via tracing edges. Computer-Aided Design 37(13) (2005) 1412-1424

19. Kim, D., Kim, D.S.: Region-expansion for the Voronoi diagram of 3D spheres. Computer-Aided Design 38(5) (May 2006) 417-430

20. Kim, D.S., Kim, D., Cho, Y., Sugihara, K.: Quasi-triangulation and interworld data struction in three dimensions. Computer-Aided Design 38(7) (2006) 808-819

21. Edelsbrunner, H., Kirkpatrick, D.G., Seidel, R.: On the shape of a set of points in the plane. IEEE TRANSACTIONS ON INFORMATION THEORY IT-29(4) (1983) 551-559

22. Edelsbrunner, H., Mücke, E.P.: Three-dimensional alpha shapes. ACM Transactions on Graphics 13(1) (January 1994) 43-72

23. Edelsbrunner, H.: The union of balls and its dual shape. Discrete \& Computational Geometry 13 (1995) 415-440

24. Kim, D.S., Seo, J., Kim, D., Ryu, J., Cho, C.H.: Three-dimensional beta shapes. Computer-Aided Design 38(11) (2006) 1179-1191

25. Halperin, D., Overmars, M.H.: Spheres, molecules, and hidden surface removal. In: Proceedings of the 10th ACM Symposium on Computational Geometry. (1994) $113-122$

26. Berman, H., Westbrook, J., Feng, Z., Gilliland, G., Bhat, T., Weissig, H., Shindyalolv, I., Bourne, P.: The protein data bank. Nucleic Acids Research 28 (2000) 235-242

27. RCSB: Protein Data Bank Homepage http://www.rcsb.org/pdb/.

28. Seidl, T., Kriegel, H.P.: Solvent accessible surface representation in a database system for protein docking. In: Proceedings of the 3rd International Conference on Intelligent Systems for Molecular Biology (ISMB'95), AAAI Press (1995) 350-358 